\title{
A Comparative Template for Reliable Requirement Specification
}

\author{
Sandeep Kumar Nayak \\ Dept. of Comp. Sc. \& Application \\ Integral University \\ Lucknow, India
}

\author{
Dr. Raees Ahmad Khan \\ Dept. of Information Technology \\ B.B.A University \\ Lucknow, India
}

\author{
Dr. Md.Rizwan Beg \\ Dept. Comp. Sc. \& Engg. \\ Integral University \\ Lucknow, India
}

\begin{abstract}
For documenting the groundwork pitch of requirements, many types of requirement specification template are available containing some specific requirement parameter which eventually recites the root requirement for constructing the design, but they are not good enough to make the template reliable requirement specification. In this paper a reliable requirement specification template has been proposed having some reliable parameter which will assess the reliability of the individual requirement before finalizing the requirement documentation which reflects the reliability of requirements at early stage of software development.
\end{abstract}

\section{Keywords}

Software Reliability, requirement specification

\section{INTRODUCTION}

Many years of the effort have gone into study of hardware reliability particularly in the area of complex electronic assemblies; however the research scholars and practitioners are giving attention to software reliability estimation as the major research area since last two decades. [3] Since the software reliability becomes the most important characteristics regarding computer system so it is necessary to measure and manage the reliability of the computer software, for that purpose many software reliability models have been proposed for the study of software reliability but as we know that most of the models are made for estimating reliability at testing phase which comes into later stage of Software Life Cycle (SLC). The failure of software depends on rooted defects that didn't come into face at early stage of requirement gathering which carried on in the further phases of development and testing and these defects will greatly affect the reliability of the software.

If the reliable requirements are gathered at initial stage then the probability of software failure (program behavior doesn't meet the user's requirement within the given time of execution i.e. failure totally depends on the leakage of user oriented concept) will certainly be decrease specifically. For giving weightage to early Requirement Suzanne and James Robertson proposed a 'Quality Gateway to find requirements related defects as early as they can be identified and hence prevent them from being incorporated in the design and implementation.' For that purpose they have suggested Volere specification template for software specification $[1,2]$

\section{WHY SHOULD A REQUIREMENT SPECIFICATION BE RELIABLE?}

It is suggested to use a proper technique to make requirement documentation unambiguous, complete and stable sothat the designer may conclude with concrete structure for software development. A good and reliable requirement specification may be helpful to recognize:
- Customer's precise description for what they actually desire to obtain.

- Software Provider understands what the customer accurately needs.

- Objective to develop a standard requirements specification outline.

- Objective to develop a requirements specification quality checklist.

A good and reliable requirement specification avoids the redesigning of the predesigned structure of the software and also retesting for fault and failure. The proposed reliable requirement specification will avoid the review of the requirement specification because it already makes itself a reliable specification.

Benefits through good and reliable requirement template:

$>$ A set up of acceptance between the customers and the suppliers about functional activities to be performed by the software.

$>$ Decrease the development time, effort and cost.

$>$ Expected augment in the quality and reliability of software to be made.

$>$ Develop a platform for estimating expenditure and plan and facilitate validation and verification.

$>$ Strongly assist the further enhancement of the finished development.

\section{FEATURES OF A RELIABLE REQUIREMENT}

Requirement may be speak out reliable on the basis of some features which qualifies each and every requirement. These features are the standards for the individual requirement.

\subsection{Completeness}

Requirements must be precisely all possible state of affairs that will be encountered and the system's capability to successfully address them.

\subsection{Consistency}

Requirement capability functions and performance levels or confidence levels must be compatible and the required quality features like correctness and trustworthiness should not counteract those capability functions. 
Fig 1. Volere Template Requirement Specification

Requirement \#: Unique id for individual requirement
Requirement Type: What Type of requirement is
Event/Use Case\#: List of events/use cases that need this requirement

Description: A one sentence statement of intention of the requirement

Rationale: A justification of the requirement

Sources: Who raised the requirement

Fit Criterion: A measurement of the requirement such that it is possible to test if the solution matches the original requirement

Customer Satisfaction: Degree of stakeholder happiness if this requirement is successfully implement (Scale from $1=$ uninterested to $5=$ extremely pleased )

Dependencies: List of other requirements that have some dependency on this one
Customer dissatisfaction: Measure of stakeholder unhappiness if this requirement is not part of the final product (Scale from $1=$ hardly matters to $5=$ extremely displeased)

Conflicts: Other requirements that cannot be implemented if this one is

Supporting Materials: Pointers to documents that illustrate and explain this requirement

History: Creation, changes

\subsection{Modifiable}

The logical and hierarchical formation of requirements should facilitate any required modifications. Related requirements should exist in group and isolates form unrelated requirements sothat modification may easier.

\subsection{Rank of requirement}

Individual requirements should arrange in hierarchy according to stability, security, perceiving ease or difficulty of implementation or other parameter that helps in the design of that and subsequent documents.

\subsection{Verifiable}

A verifiable requirement must be consistent from one level of abstraction to another. Requirement attributes must be split in FACT \& DIMENSION table format so to make it conclusive and they must be verified through strength and weakness of presented attributes.

\subsection{Robustness}

Healthy requirement will help developer to build up an accurate architecture for proposed system.

\subsection{Portability}

Transportation of requirement must refer different knowledge workers to come up with one motive of development.

\subsection{Functional Capable}

Requirements must be functionally capable to construct the design of genuine and applicable functional part of the system.

\subsection{Unambiguous}

Requirement documenting is about to communication so the written text must be dependent on real expressed words of user and must convey the same. There are two features for requirement ambiguity-

(a) Writer Ambiguity- not having a clear interpretation of the requirements (b) Reader Ambiguity- requirements that, as written, can be interpreted in more than one way.

\section{RELIABILITY PARAMETERS OF THE TEMPLATE}

Some stringent steps must be taken to make requirement reliable with the help of some of the parameters mentioned below which will characterize the appropriate reliable requirement for the development of reliable software.

\subsection{Severity Level}

It is related to technical aspect of the software product. Severity Level reflects how terrible the individual requirement is for the system. It is decided as per the risk assessment of the customer. The requirement quality assurance analyst use to make a decision on severity level.

Critical: This type of requirement defect is extremely severe and may capable to halt the entire system. If this requirement is implemented then the software application will refuse to execute or crooked of data.

Major: This type of requirement defect is also a severe and may capable to halt some functions of the system with serious degradation in performance. Although this is also a failure, but some operations carry on at a lower rate of performance.

Minor: This type of requirement defect is not so severe but caused a low-level disruption of the system or malfunctioning of some operations. This kind of disruption or nonavailability of some functionality can be acceptable for a limited period. This type of disruption may cause for failure, but it continues to operate for short span of time at a lower rate of performance.

Cosmetic: This type of requirement defect is one that mainly related to the appearance or the layout of the data which has no risk of corrupting the statistics and incorrect values. Since it converse about the headline, flag, marker and Colors so it has no actual effect on the operations and may carry on operating with system without any degradation in performance.

\subsection{Confidence Level}

The confidence level of the requirement tells about how certain the requirement may be. It is expressed as a proportion and represents how often the true fraction of the original one and also how likely the quantitative or qualitative range is to contain

by the parameter is determined by the confidence level. As the confidence level desirous to increase confidence range will certainly be widen. Thus, confidence range of the requirement is used to indicate the reliability of an estimated requirement. [6]

\subsection{Rank of Requirement}

Rank of the requirement will represent the status of the requirement whether it is: 
Essential: The requirement which is necessary for the functionality of the software product that means it will play a vital role for the systematic operation of the product.

Optional: Some requirements are not essential but they are optional which may not affect on the major operations of the system they may be treated as helping or elective requirement.

Conditional: Some of the requirements are the conditional which used to depend on other requirements. It may be essential or optional.

\section{PROPOSED WORK}

Fig.1 Volere Template for Requirement Specification [2] is meant for the good and complete software requirement specification but through study it has been analyzed that it may not be the reliable requirement specification for further treatment in software development so through this paper an effort of making this template reliable, has been placed. In fig. 2 four major reliability parameters has been introduced which will help to make the Volere template a reliable describe in above section-4. Through this we may say that the individual requirement taken within the template will be best fit into concrete world without having platonic ideas.

\section{CONCLUSION}

Requirement severity level will be assessed through the fit criteria of the requirement. If the individual requirement solution is not reaching the original requirement then there are some defects in the requirement and how they affect the system will be review through the severity level of the requirement.

Rank of the requirement will qualify the confidence level of the requirement through mentioning the status of the requirement. Confidence level of the requirement will be assessed through the severity level after classifications of the requirement it will be clear that if Severity Level is:

Cosmetic: Requirements are fully permissible with no degradation in performance.

\section{Requirement \#: Unique id for \\ individual requirement \\ Requirement Type: What Type of requirement is \\ Event/Use Case\#: List of events/use cases that need this requirement \\ Description: A one sentence statement of intention of the requirement \\ Rationale: A justification of the requirement \\ Sources: Who raised the requirement \\ Fit Criterion: A measurement of the requirement such that it is possible to test if the solution matches the original requirement}

Customer Satisfaction: Degree of stakeholder happiness if this requirement is successfully implement (Scale from $1=$ uninterested to $5=$ extremely pleased )

Dependencies: List of other requirements that have some dependency on this one
Customer dissatisfaction: Measure of stakeholder unhappiness if this requirement is not part of the final product (Scale from $1=$ hardly matters to $5=$ extremely displeased)

Conflicts: Other requirements that cannot be implemented if this one is

Supporting Materials: Pointers to documents that illustrate and explain this requirement

History: Creation, changes

Severity Level: Level of severity if this requirement is consider ( severity level of requirement $\rightarrow$ Critical or Major or Minor or Cosmetic)

Reliability Characteristics of Requirement: Quantitative or Qualitative reliability value of the requirement

Rank of the Requirement: Essential, Optional, Conditional

Confidence Level: This requirement proportion to original one (permissibility extent of requirement)

Fig 2. Reliable Requirement Specification Template

template for requirement specification. In this template the major proposed parameter is Reliability characteristics of Requirement which will be assessed in terms of Quantitative or Qualitative value, for qualitative value we will have to consider the weightage/scaling of individual requirement and for quantitative value we have find out the failure probability of same requirement then by the definition of reliability, $\mathrm{R}(\mathrm{t})=1-\mathrm{F}(\mathrm{t})$;

$F(t)$ is the probability of failure during time t. $[3,4]$ Here,

The reliability of the individual requirement will be

$\mathrm{R}(\mathrm{t})=1-\{\operatorname{Pr}(\mathrm{f}(\mathrm{Ri}))\}$

Here,

$\operatorname{Pr}(\mathrm{f}(\mathrm{Ri})$ ) is the failure probability of the individual requirement $(\mathrm{Ri})$; and this failure probability may be assess through severity class, rank of the requirement and confidence level defined within the template which is
Minor: Requirements are permissible for short span of time with some degradation in performance.

Major: Few requirements may permissible at lower rate of performance.

Critical: Requirements are not permissible because it halted the system.

In this paper we are trying to deliver a concrete reliable requirement so that the reliability of the software can be estimated at the early stage of the software development, through this attempt the accumulated cost at the testing phase will certainly be decreased and more reliable software product will produce. 


\section{REFERENCES}

[1] Suzanne Robertson, James Robertson, "Reliable Requirements through the Quality Gateway", $10^{\text {th }}$ International Workshop on Database and Expert System Application, September 1999, pp. 357-363.

[2] Suzanne Robertson, James Robertson, "Volere Requirements Specification Template" Edition 11February 2006, Volere Requirement Resources http://www.volere.co.uk

[3] Shiyi Xu "An Accurate Model of Software Reliability", 13th IEEE International symposium on Pacific Rim Dependable Computing, Dec. 2007, pp.77-84
[4] Shiyi Xu, "Reconsideration of Software Reliability Measurements", $16^{\text {th }}$ IEEE Asian Test Symposium, 2007, pp. 159-162

[5] "Difference between Priority and Severity" and "Understanding Software Defects, their Severity \& Priority

Resolution' http://www.softwaretestinggenius.com/

[6] "Confidence-interval" http://en.wikipedia.org/wiki/Confidence_interval 